\title{
Stereotactic body radiation therapy versus fractionated radiation therapy for early-stage bronchopulmonary carcinoid
}

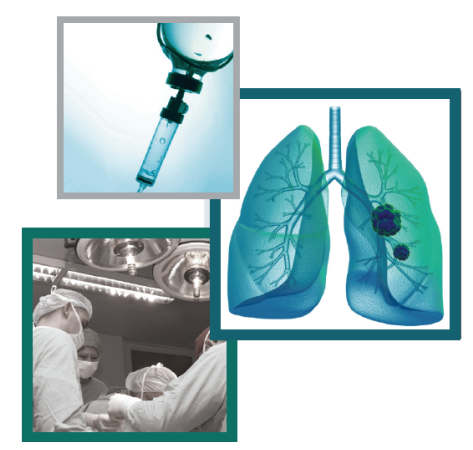

\author{
Rodney E Wegner*,1, Stephen Abel ${ }^{1}$, Zachary D Horne ${ }^{1}$, Shaakir Hasan ${ }^{1}$, Athanasios \\ Colonias $^{1}$ \& Vivek Verma ${ }^{1}$ \\ ${ }^{1}$ Allegheny Health Network Cancer Institute, Division of Radiation Oncology, 320 E. North Ave, Pittsburgh, PA 15212, USA \\ *Author for correspondence: Tel.: +1 412359 3400; Fax: +1 412359 3171; Rodney.wegner@ahn.org
}

\begin{abstract}
Aim: To compare trends and outcomes in early stage bronchopulmonary carcinoid (BPC) tumors treated nonoperatively with conventionally fractionated radiotherapy (CFRT) and stereotactic body radiotherapy (SBRT). Methods/materials: We queried the National Cancer Database for primary (typical) BPC staged CT1-2NOM0 and treated nonsurgically with lung-directed radiation and $\geq 1$ month of follow-up. Odds ratios were used to predict likelihood of SBRT treatment and multivariable Cox regression determined predictors of survival. Results: Out of 154 patients, 84 (55\%) were treated with SBRT and the remainder were treated with CFRT. Although SBRT use was 0\% from 2004 to 2007, it varied from 50 to $70 \%$ per year thereafter. Propensity-matched Kaplan-Meier analysis revealed improved survival with lung SBRT (median: 66 vs 58 months; $\mathrm{p}=0.034$ ). Conclusion: SBRT for early stage, primary BPC has increased over time and was associated with higher survival than CFRT.
\end{abstract}

First draft submitted: 13 February 2019; Accepted for publication: 27 June 2019; Published online:

21 August 2019

Keywords: bronchopulmonary carcinoid • lung cancer • NSCLC • SBRT

\section{Background}

Low-grade neuroendocrine tumors of the lung, traditionally referred to as typical pulmonary carcinoids, are rare and usually follow an indolent clinical course [1-3]. For patients able to undergo surgery, resection remains the preferred management and standard of care, resulting in excellent local control (>95\%) [4-6]. Often, these tumors occur in older patients not amenable to surgical resection due to medical comorbidities. In this situation, radiation is considered a reasonable alternative [7].

In more recent years, stereotactic body radiotherapy (SBRT) has emerged as an efficacious method to deliver ablative doses of radiation to lung tumors, typically in medically inoperable patients with non-small-cell lung cancer (NSCLC) $[8,9]$. In this setting, as compared with conventionally fractionated radiation therapy (CFRT), SBRT has shown to have improved outcomes and is preferred in appropriately selected patients, resulting in increased utilization over the past 10-15 years [10].

However, the experience with SBRT for pulmonary carcinoids is much more limited, being reported in only a few small series [11,12]. SBRT for these neoplasms may be particularly beneficial owing to the relatively radioresistant nature [13]. As such, we sought to use the National Cancer Database (NCDB) to examine trends and outcomes with the use of SBRT as compared with CFRT.

\section{Methods}

The methods for analyzing the NCDB have been previously outlined [14-16]. This retrospective review comprised de-identified data from the NCDB, therefore, an IRB waiver was permitted. Overseen by the American Cancer Society and American College of Surgeons, the NCDB encompasses an estimated $70 \%$ of annual cancer cases in the USA. The American College of Surgeons and the Commission on Cancer have not verified and are not responsible for the analytic or statistical methodology employed, or the conclusions drawn from these data by the investigator.

Future $\because$ Medicine 


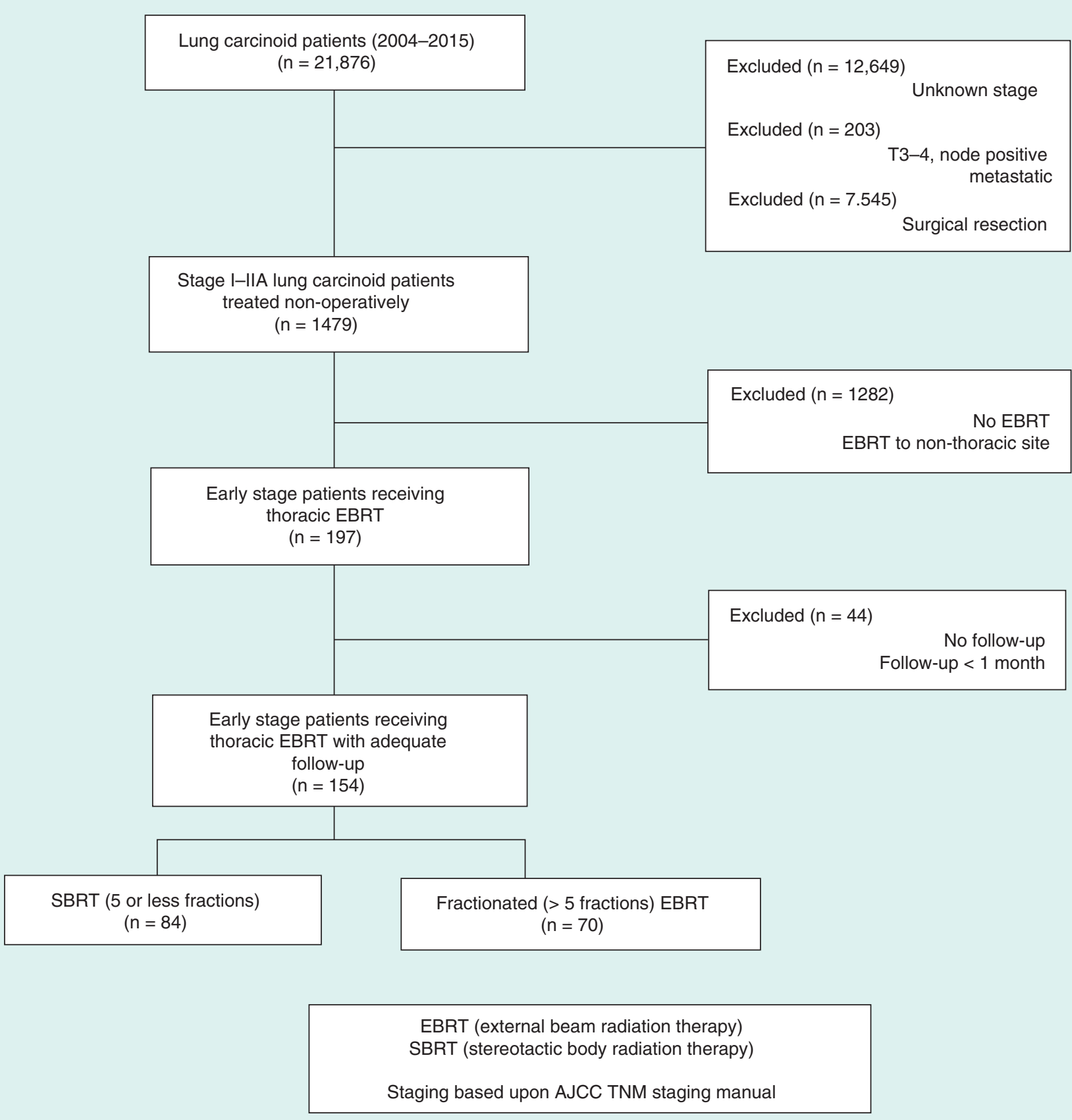

Figure 1. CONSORT diagram showing patient selection.

AJCC: American Joint Committee on Cancer; EBRT: External beam radiation therapy; SBRT: Stereotactic body radiotherapy; TNM: Tumor, nodes, metastasis.

Patients with documented clinical stage T1-2N0 typical pulmonary carcinoid who were treated nonoperatively with radiation to the lung were included in this study (atypical carcinoids were not included in this study due to the small number treated with SBRT or even CFRT). Figure 1 is a CONSORT diagram outlining the cohort selection criteria. We excluded patients not receiving radiation, treated with surgery, or having unknown/inadequate (i.e., $<1$ month) follow-up to account for immortal time bias.

Race was categorized as Caucasian, African American or other. The Charlson/Deyo comorbidity index quantified the degree of comorbidities [17]. Socioeconomic data in the patients' residence census tract were divided into quartiles 
based upon the percentage of persons with less than a high school education and median household income. Facility type was grouped according to the Commission on Cancer accreditation category. Locations were described based on data provided by the US Department of Agriculture Economic Research Service. Insurance status is documented in the NCDB as it appears on the admission page.

Data were analyzed using Medcalc Version 18 (Ostend, Belgium). Summary statistics are presented for discrete variables. Chi-squared tests compared sociodemographic treatment and tumor characteristics between the treatment groups. Overall survival is recorded in the NCDB in months from time of diagnosis to death or censored at last contact. Kaplan-Meier curves were used to calculate cumulative probability of survival [18]. Of note, outcomes like local failure, regional failure, distant failure as well as salvage therapy are not included in the NCDB. Log-rank statistics were used to test for a statistically significant difference in the cumulative proportions across groups. A multivariable survival analysis was conducted using a Cox proportional hazards model [11]. Factors significant on univariable analysis were entered using a stepwise elimination process. Adjusted hazard ratios and $95 \%$ confidence intervals are reported, using an alpha level of 0.05 to indicate statistical significance.

To account for indication biases associated with lack of randomization, propensity score-matching was applied to the multivariable survival analysis [19]. Multivariable logistic regression was used to calculate a propensity score indicative of conditional probability of receiving SBRT. The propensity model included observable variables associated with treatment selection on multivariable logistic regression. Patients were then matched based on propensity score and analyzed using Kaplan-Meier curves. The assumption of balance was further validated by stratifying the data into propensity score-based quintiles, and confirming that the difference in propensity score mean per quintile was less than 0.10 .

\section{Results}

We identified 154 patients in the NCDB between 2004 and 2014 meeting the above eligibility criteria, of whom $84(55 \%)$ were treated with SBRT. Table 1 outlines the baseline characteristics of the entire cohort. The median age was 74 years (interquartile range: 65-81), most patients (76\%) were stage T1 and very few received systemic therapy (6\%). Patients with T2 stage and receipt of chemotherapy were more likely to be treated with CFRT (Table 2). For patients treated with radiation, the rate of SBRT use was $0 \%$ from 2004 to 2007, thereafter, it varied from 50 to $70 \%$ per year. The median CFRT dose was 54 Gy (interquartile range: 50-60) in 24 fractions (interquartile range: 5-33) and the median SBRT dose was 50 Gy (interquartile range: 50-55 Gy) in four fractions (interquartile range: 4-5).

Median follow-up was 30 months (1-107 months) for the entire cohort. Multivariable analysis identified age $<74$ years, SBRT technique, female sex and more recent year of diagnosis as predictive of improved survival (Table 3). Unadjusted Kaplan-Meier analysis demonstrated improved overall survival in patients receiving SBRT compared with patients receiving CFRT (median: 65 vs 58 months, $\mathrm{p}=0.0184$ ), as shown in Supplementary Figure 1. Propensity score was generated and incorporated education level and income level following multivariable logistic regression. Fifty-seven pairs were generated based on an exact propensity score match. Propensity-matched Kaplan-Meier analysis revealed improved survival with lung SBRT, median overall survival of 66 months compared with 58 months, $\mathrm{p}=0.0344$ (Figure 2). We also ran the propensity-matched Kaplan-Meier analysis including only T1 patients with survival benefit still seen with SBRT (median overall survival of 66 months compared with 58 months, $\mathrm{p}=0.0171$ ).

\section{Discussion}

Pulmonary carcinoid tumors are relatively rare and are second to gastrointestinal sites in terms of location [12]. Surgical resection remains the preferred treatment, yielding local control rates $>95 \%$ and allowing for sampling of nodal tissue at the time of intervention [20]. As evidenced by the population presented here (median age 74 years), these patients are often older and either cannot tolerate surgical intervention or refuse it. In those instances, radiation offers a reasonable alternative definitive treatment, extrapolating from many years of use of CFRT in NSCLC [21]. Over the past 10-15 years, lung SBRT has emerged as a highly effective and safe alternative to surgery, typically in nonoperable NSCLC patients [8-10,22]. Naturally, the use of SBRT for NSCLC and other lung tumors has sharply expanded over the past $10+$ years [9].

The use of SBRT in pulmonary carcinoid, however, has been reported sparingly. A small case series from Yale reviewed outcomes in four patients with pulmonary carcinoid, all treated with SBRT in a safe and effective manner, lending some support to SBRT as an alternative in patients that do not get treated surgically [13]. Of note, in this 
Table 1. Patient demographics and clinical characteristics at baseline $(n=154)$.

Characteristic

Sex:

- Male

- Female

Race:

- White

- African-American

- Other

Comorbidity score:

$-0$

$-1$

$-\geq 2$

Insurance:

- Not insured

- Private payer

- Government

- Unrecorded

Education (\%):

$-\geq 29$

$-20-28.9$

$-14-19.9$

$-<14$

Treatment facility type:

- Community cancer program

- Comprehensive community cancer program

- Academic/research program

Treatment facility location:

- Metro

- Urban

- Rural

Income, US dollars:

$-<30,000$

$-30,000$ to 35,000

$-35,000$ to 45,999

$->46,000$

Distance to treatment facility (miles):

$-\leq 10$

$->10$

Age distribution (years):

$-\leq 74$

$->74$

Year of diagnosis:

-2004-2006

-2007-2009

- 2010-2012

-2013-2014

T stage:

$-1$

$-2$

Chemotherapy:

- No

- Yes
Number (\%)

43 (28)

$111(72)$

$139(90)$

14 (9)

1 (1)

97 (63)

37 (24)

20 (13)

$0(0)$

30 (19)

122 (79)

2 (2)

22 (14)

39 (25)

60 (39)

33 (22)

2 (1)

69 (45)

82 (54)

$121(80)$

25 (17)

4 (3)

30 (19)

37 (24)

44 (29)

43 (28)

79 (51)

75 (49)

81 (53)

73 (47)

4 (3)

14 (9)

62 (40)

74 (48)

117 (76)

37 (24)

144 (94)

$10(6)$ 
Table 2. Comparative use of stereotactic body radiotherapy by baseline characteristics in patients receiving lung radiation.

\begin{tabular}{|c|c|c|c|c|c|}
\hline Characteristic & CFRT $(n=70)(\%)$ & SBRT $(n=84)(\%)$ & Odds ratio & $95 \% \mathrm{Cl}$ & p-value \\
\hline \multicolumn{6}{|l|}{ Sex: } \\
\hline - Male & $20(29)$ & $23(27)$ & 1 & Reference & \\
\hline - Female & $50(71)$ & $61(73)$ & 1.06 & $0.52-2.15$ & 0.87 \\
\hline \multicolumn{6}{|l|}{ Race: } \\
\hline - White & $65(93)$ & $74(88)$ & 1 & Reference & \\
\hline - African American & $5(7)$ & $9(11)$ & 1.58 & $0.50-4.96$ & 0.43 \\
\hline - Other & $0(0)$ & $1(1)$ & 2.64 & $0.11-65.87$ & 0.55 \\
\hline \multicolumn{6}{|l|}{ Comorbidity score: } \\
\hline-0 & $43(61)$ & $54(64)$ & 1 & Reference & \\
\hline-1 & $19(27)$ & $18(21)$ & 0.75 & $0.35-1.61$ & 0.47 \\
\hline$-\geq 2$ & $8(12)$ & $12(15)$ & 1.19 & $0.45-3.18$ & 0.72 \\
\hline \multicolumn{6}{|l|}{ Age (years): } \\
\hline$-\leq 74$ & $38(54)$ & $43(51)$ & 1 & Reference & \\
\hline$->74$ & $32(46)$ & $41(49)$ & 1.13 & $0.60-2.14$ & 0.70 \\
\hline \multicolumn{6}{|l|}{ Insurance: } \\
\hline - Private payer & $18(26)$ & $12(14)$ & 1 & Reference & \\
\hline - Government & $51(74)$ & $71(86)$ & 2.09 & $0.93-4.71$ & 0.08 \\
\hline \multicolumn{6}{|l|}{ Education: } \\
\hline$-\geq 29 \%$ & $13(19)$ & $9(11)$ & 1 & Reference & \\
\hline$-20-28.9$ & $15(21)$ & $24(29)$ & 2.31 & $0.80-6.72$ & 0.12 \\
\hline$-14-19.9$ & $27(39)$ & $33(39)$ & 1.77 & $0.66-4.75$ & 0.26 \\
\hline$-<14$ & $15(21)$ & $18(21)$ & 1.73 & $0.58-5.16$ & 0.32 \\
\hline \multicolumn{6}{|l|}{ Facility type: } \\
\hline - Community cancer program & $1(1)$ & $1(1)$ & 1 & Reference & \\
\hline - Comprehensive cancer program & $31(45)$ & $38(45)$ & 1.23 & $0.07-20.40$ & 0.89 \\
\hline - Academic/research program & $37(54)$ & $45(54)$ & 1.22 & $0.07-20.12$ & 0.89 \\
\hline \multicolumn{6}{|l|}{ Facility location: } \\
\hline - Metro & $59(86)$ & $62(77)$ & 1 & Reference & \\
\hline - Urban & $9(13)$ & $16(20)$ & 1.69 & $0.69-4.12$ & 0.25 \\
\hline - Rural & $1(1)$ & $3(3)$ & 2.85 & $0.29-28.22$ & 0.37 \\
\hline \multicolumn{6}{|l|}{ Income (USD): } \\
\hline$-<30,000$ & $11(16)$ & $19(23)$ & 1 & Reference & \\
\hline$-30,000-35,000$ & $16(23)$ & $21(25)$ & 0.76 & $0.28-2.04$ & 0.59 \\
\hline$-35,000-45,999$ & $17(24)$ & $27(32)$ & 0.92 & $0.35-2.40$ & 0.86 \\
\hline$->46,000$ & $26(37)$ & $17(19)$ & 0.38 & $0.14-0.99$ & 0.05 \\
\hline \multicolumn{6}{|l|}{ T stage: } \\
\hline-1 & $45(64)$ & $72(86)$ & 1 & Reference & \\
\hline-2 & $25(36)$ & $12(14)$ & 0.30 & $0.13-0.66$ & 0.0026 \\
\hline \multicolumn{6}{|l|}{ Distant to facility: } \\
\hline$-\leq 10$ miles & $36(51)$ & $43(51)$ & 1 & Reference & \\
\hline$->10$ miles & $34(49)$ & $41(49)$ & 1.01 & $0.54-1.90$ & 0.98 \\
\hline \multicolumn{6}{|l|}{ Received chemotherapy: } \\
\hline- No & $62(89)$ & $82(98)$ & 1 & Reference & \\
\hline - Yes & $8(11)$ & $2(2)$ & 0.19 & $0.04-0.92$ & 0.0393 \\
\hline \multicolumn{6}{|l|}{ Year of diagnosis: } \\
\hline$-2004-2006$ & $4(6)$ & $0(0)$ & 1 & Reference & \\
\hline$-2007-2009$ & $7(10)$ & $7(8)$ & 9.00 & $0.41-198.22$ & 0.16 \\
\hline$-2010-2012$ & $27(39)$ & $35(42)$ & 11.62 & $0.60-225.09$ & 0.10 \\
\hline$-2013-2015$ & $32(45)$ & $42(50)$ & 11.77 & $0.61-226.50$ & 0.10 \\
\hline
\end{tabular}


Table 3. Multivariable Cox regression for predictors of survival in patients with pulmonary carcinoid treated with radiation therapy.

\begin{tabular}{|c|c|c|}
\hline Characteristic & HR $(95 \% \mathrm{Cl})$ & $\mathrm{p}$-value \\
\hline \multicolumn{3}{|l|}{ Age (years): } \\
\hline$-\leq 74$ & Reference & \\
\hline$->74$ & $2.06(1.16-3.67)$ & 0.0134 \\
\hline \multicolumn{3}{|c|}{ Radiation technique: } \\
\hline - CFRT & Reference & \\
\hline - SBRT & $0.48(0.28-0.85)$ & 0.0110 \\
\hline \multicolumn{3}{|l|}{ Sex: } \\
\hline - Male & Reference & \\
\hline - Female & $0.51(0.27-0.94)$ & 0.0305 \\
\hline \multicolumn{3}{|c|}{ Year of treatment: } \\
\hline$-2004-2006$ & Reference & \\
\hline$-2007-2009$ & $0.36(0.14-0.95)$ & 0.0396 \\
\hline$-2010-2012$ & $0.52(0.16-1.66)$ & 0.2723 \\
\hline$-2013-2014$ & $0.52(0.27-1.04)$ & 0.0628 \\
\hline
\end{tabular}

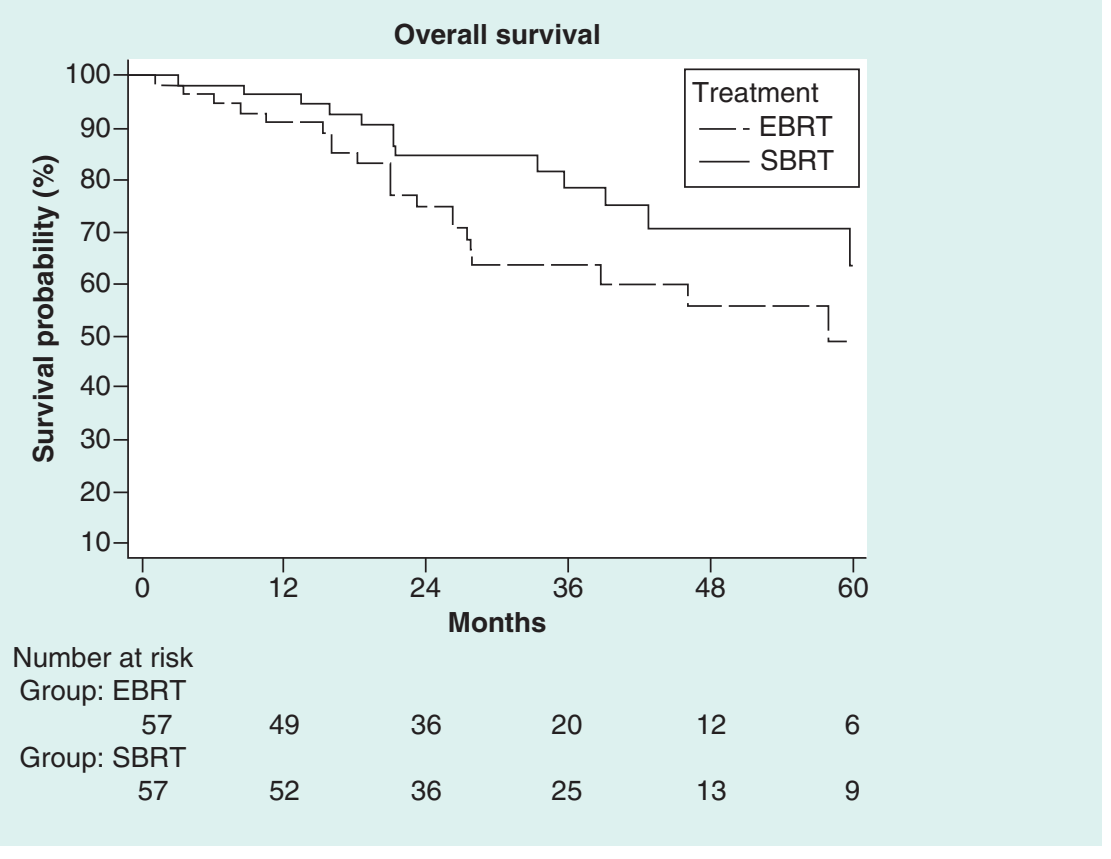

Figure 2. Overall survival comparison between patients with typical carcinoid treated with either conventionally fractionated radiation therapy or stereotactic body radiotherapy. Median survival was 66 months compared with 58 months, in favor of SBRT, $\mathrm{p}=0.0344$.

EBRT: External beam radiation therapy; SBRT: Stereotactic body radiotherapy.

analysis we did not directly compare surgical resection to SBRT, instead focusing on the difference in radiation techniques. We did show, after propensity matching that the patients treated with SBRT had better survival, suggesting a benefit compared with CFRT. One must keep in mind that patients with T2 tumors and those receiving chemotherapy were more likely to receive CFRT. Granted, the receipt of chemotherapy for stage T1-2N0 typical pulmonary carcinoid does not follow recognized guidelines, and likely implies some other undocumented clinicopathologic factors contributing to worse outcome. In addition, the tumor location was not reported and centrally located tumors may not be as amenable to SBRT and are more likely to cause symptoms or potential patient demise [23-25]. Another consideration is the ability of SBRT to deliver a higher biologically effective dose, 
which one would expect to result in better outcomes given the relative radioresistance of carcinoids [13]. Taking all of the above into account, SBRT may be the preferred technique in nonoperative candidates with pulmonary carcinoid.

The limitations of the current study include its retrospective nature and inherent selection bias as a result. The NCDB also lacks critical data as it relates to operability such as performance status, extent of work up, pulmonary function testing and tumor location as mentioned above. The NCDB also does not record outcomes outside of survival, such as local failure, regional failure and any salvage therapy, which are all important and interrelated to survival in this patient population.

\section{Conclusion}

This analysis of the NCDB shows a steady increase in the use of SBRT for typical pulmonary carcinoids managed in a nonoperative fashion. There was a survival benefit seen with SBRT compared with CFRT, which persisted on propensity matching. In light of the sharply expanding use of SBRT over CFRT for other early stage lung tumors, SBRT may be considered as the preferred option for T1-2N0 pulmonary carcinoids.

\section{Summary points}

- Historically, early stage bronchopulmonary carcinoid has been treated with surgery or conventionally fractionated radiotherapy (CFRT).

- Stereotactic body radiotherapy (SBRT) has emerged as the preferred treatment approach in inoperable cases of non-small-cell lung cancer; however, data in cases of primary typical lung carcinoid are limited.

- We performed a retrospective review of a large, contemporary database, analyzing 154 patients with early stage lung carcinoid treated with CFRT $(n=70)$ or SBRT $(n=84)$ from 2004 to 2014.

- SBRT use was $0 \%$ from 2004 to 2007 , but increased to 50 to $70 \%$ per year thereafter and overall survival was longer (median: 66 months versus 58 months; $p=0.034$ ) in those receiving SBRT compared with CFRT.

- SBRT may be considered as the preferred treatment option for nonoperative early stage patients.

\section{Supplementary data}

To view the supplementary data that accompany this paper please visit the journal website at: www.futuremedicine.com/doi/full/ 10.2217/Imt-2019-0003

\section{Author contributions}

RE Wegner performed project conception and design, statistical analysis and manuscript drafting. S Abel, ZD Horne and A Colonias did manuscript revisions. S Hasan performed statistical analysis and manuscript revisions. $V$ Verma performed manuscript revision, project conception and design.

\section{Financial \& competing interests disclosure}

The authors have no relevant affiliations or financial involvement with any organization or entity with a financial interest in or financial conflict with the subject matter or materials discussed in the manuscript. This includes employment, consultancies, honoraria, stock ownership or options, expert testimony, grants or patents received or pending, or royalties.

No writing assistance was utilized in the production of this manuscript.

\section{Open access}

This work is licensed under the Attribution-NonCommercial-NoDerivatives 4.0 Unported License. To view a copy of this license, visit http://creativecommons.org/licenses/by-nc-nd/4.0/

\section{References}

Papers of special note have been highlighted as: • of interest; $\bullet \bullet$ of considerable interest

1. Buschi AJ, Smith TH. Bronchial carcinoid. VA Med. Mon. 102(12), 1027-1031 (1976).

2. Gustafsson BI, Kidd M, Chan A, Malfertheiner MV, Modlin IM. Bronchopulmonary neuroendocrine tumors. Cancer 113(1), 5-21 (2008).

3. Hemminki K, Li X. Incidence trends and risk factors of carcinoid tumors: a nationwide epidemiologic study from Sweden. Cancer 92(8), 2204-2210 (2001). 
4. Carretta A, Ceresoli GL, Arrigoni G et al. Diagnostic and therapeutic management of neuroendocrine lung tumors: a clinical study of 44 cases. Lung Cancer 29(3), 217-225 (2000).

5. Ferguson MK, Landreneau RJ, Hazelrigg SR et al. Long-term outcome after resection for bronchial carcinoid tumors. Eur. J. Cardiothorac. Surg. 18(2), 156-161 (2000).

6. Filosso PL, Oliaro A, Ruffini E et al. Outcome and prognostic factors in bronchial carcinoids: a single-center experience. J. Thorac. Oncol. 8(10), 1282-1288 (2013).

7. Mackley HB, Videtic GM. Primary carcinoid tumors of the lung: a role for radiotherapy. Oncology (Williston Park) 20(12), 1537-1545, 1549 (2006).

8. Timmerman R, Paulus R, Galvin J et al. Stereotactic body radiation therapy for inoperable early stage lung cancer. JAMA 303(11), 1070-1076 (2010).

9. Hasan S, Colonias A, Mickus T, Vandeusen M, Wegner RE. Image-based management of empiric lung stereotactic body radiotherapy (SBRT) without biopsy: predictors from a 10-year single institution experience. Thorac. Cancer 9(6), 699-706 (2018).

10. Haque W, Verma V, Polamraju P, Farach A, Butler EB, Teh BS. Stereotactic body radiation therapy versus conventionally fractionated radiation therapy for early stage non-small-cell lung cancer. Radiother. Oncol. 129(2), 264-269 (2018).

11. Cox DR. Regression models and life- tables. J. R. Stat. Soc. 34(2), 187-220 (1972).

12. Modlin IM, Lye KD, Kidd M. A 5-decade analysis of 13,715 carcinoid tumors. Cancer 97(4), 934-959 (2003).

-• Robust analysis of carcinoid management prior to the substantial increase in stereotactic body radiotherapy utilization.

13. Colaco RJ, Decker RH. Stereotactic radiotherapy in the treatment of primary bronchial carcinoid tumor. Clin. Lung Cancer. 16(2), e11-e14 (2015).

14. Hasan S, Renz P, Turrisi A, Colonias A, Finley G, Wegner RE. Dose escalation and associated predictors of survival with consolidative thoracic radiotherapy in extensive stage small cell lung cancer (SCLC): A National Cancer Database (NCDB) propensity-matched analysis. Lung Cancer 124, 283-290 (2018).

15. Hasan S, Renz P, Wegner RE et al. Microsatellite instability (MSI) as an independent predictor of pathologic complete response (PCR) in locally advanced rectal cancer: A National Cancer Database (NCDB) Analysis. Ann. Surg. doi:10.1097/SLA.0000000000003051 (2018) (Epub ahead of print).

16. Wegner RE, Abel S, Hassan S et al. The role of adjuvant therapy for atypical bronchopulmonary carcinoids. Lung Cancer 131, 90-94 (2019).

17. Deyo RA, Cherkin DC, Ciol MA. Adapting a clinical comorbidity index for use with ICD-9-CM administrative databases. J. Clin. Epidemiol. 45(6), 613-619 (1992).

18. Meier ELKaP. Nonparametric estimation from incomplete observations. J. Am. Stat. Assoc. 53(282), 457-481 (1958).

19. D'agostino RB Jr. Propensity score methods for bias reduction in the comparison of a treatment to a non-randomized control group. Stat. Med. 17(19), 2265-2281 (1998).

20. Okoye CC, Jablons DM, Jahan TM, Kukreja J, Cardozo S, Yom SS. Divergent management strategies for typical versus atypical carcinoid tumors of the thoracic cavity. Am. J. Clin. Oncol. 37(4), 350-355 (2014).

- Overview of differential approaches in the management of typical versus atypical carcinoid.

21. Onishi H, Shirato H, Nagata Y et al. Hypofractionated stereotactic radiotherapy (HypoFXSRT) for stage I non-small cell lung cancer: updated results of 257 patients in a Japanese multi-institutional study. J. Thorac. Oncol. 2(7 Suppl 3), S94-S100 (2007).

22. Wegner RE, Ahemd N, Hasan S, Schumacher LY, Van Deusen M, Colonias A. SBRT for early stage lung cancer: outcomes from biopsy proven and empirically treated lesions. Lung Cancer Manag. 7(1), LMT01 (2018).

23. Horne ZD, Richman AH, Dohopolski MJ, Clump DA, Burton SA, Heron DE. Stereotactic body radiation therapy for isolated hilar and mediastinal non-small cell lung cancers. Lung Cancer 115, 1-4 (2018).

24. Verstegen NE, Lagerwaard FJ, Haasbeek CJ, Slotman BJ, Senan S. Outcomes of stereotactic ablative radiotherapy following a clinical diagnosis of stage I NSCLC: comparison with a contemporaneous cohort with pathologically proven disease. Radiother. Oncol. 101(2), 250-254 (2011).

25. Ahmed N, Hasan S, Schumacher L, Colonias A, Wegner RE. Stereotactic body radiotherapy for central lung tumors: finding the balance between safety and efficacy in the 'no fly' zone. Thorac. Cancer 9(10), 1211-1214 (2018). 